\title{
Practical Risk Stratification Score for Prediction of Contrast- Induced Nephropathy After Primary Percutaneous Coronary Intervention in Patients With Acute ST-Segment Elevation Myocardial Infarction
}

\author{
Sukrisd Koowattanatianchai ${ }^{\text {a }}$, Thamarath Chantadansuwan ${ }^{\text {, e }}$, Akaphol Kaladee ${ }^{\mathrm{c}}$, \\ Phichayut Phinyo ${ }^{\text {d }}$, Jayanton Patumanond ${ }^{\mathrm{d}}$
}

\begin{abstract}
Background: Contrast-induced nephropathy (CIN) is a common complication of percutaneous coronary intervention (PCI). This study aimed to develop a new risk stratification score that is simpler and more practical than the standard Mehran risk score (MRS) in prediction of CIN after primary PCI in ST-segment elevation myocardial infarction (STEMI) patients.
\end{abstract}

Methods: A prognostic prediction research with clinical risk score development was conducted. All STEMI patients who underwent primary PCI at the Central Chest Institute from June 1, 2017 to June 30, 2018 were included. Multivariable logistic regression analysis was used to identify independent predictors of CIN with a significant $\mathrm{P}$ value $<0.05$. Logistic coefficients of each predictor were used for score weighting and transformation. Predictive performance was validated and compared between newly-derived risk score and the MRS by nonparametric receiver operating characteristic (ROC) regression.

Results: A total of 217 patients, 43 (19.8\%) with CIN and 174 $(80.2 \%)$ without CIN, were included for score derivation. A total of 13 potential predictors were explored under multivariable logistic regression model and were subsequently eliminated. The new risk score was based on three final predictors which were ejection fraction of less than $40 \%$, triple-vessel disease as findings from angiogram, and the use of intra-aortic balloon pump (IABP). With only three predictor variables, the score predicted the risk of CIN with good discrimi-

Manuscript submitted August 25, 2019, accepted September 12, 2019

aDivision of Cardiology, Department of Medicine, Faculty of Medicine, Burapha University, Chonburi, Thailand

${ }^{b}$ Cardiology Department, Central Chest Institute, Nonthaburi, Thailand

'School of Health Science, Sukhothai Thammathirat Open University, Nonthaburi, Thailand

${ }^{\mathrm{d}}$ Center for Clinical Epidemiology and Clinical Statistics, Faculty of Medicine, Chiang Mai University, Chiang Mai, Thailand

${ }^{e}$ Corresponding Author: Thamarath Chantadansuwan, Department of Cardiology, Central Chest Institute of Thailand, Tiwanond Road, Mueang District, Nonthaburi 11000, Thailand. Email: dansuwan_t@yahoo.co.th

doi: https://doi.org/10.14740/cr939 native ability (area under the receiver operating characteristic curve (AuROC): 0.83, 95\% confidence interval (CI): 0.76 - 0.90) which was higher than that of the MRS (AuROC: $0.78,95 \%$ CI: $0.69-0.87$ ). The score was categorized into low-risk (positive predictive value (PPV): 9.9, 95\% CI: 5.4 - 14.4) and high-risk (PPV: 56.5, 95\% CI: 42.4 70.8) groups at the cut-off point of 2 .

Conclusions: The newly developed score was proved to have good predictive performance with fewer numbers of predictors and could be practically applied for risk stratification of CIN in STEMI patients who required emergent primary PCI.

Keywords: Prediction score; Contrast-induced nephropathy; STEMI

\section{Introduction}

One of the most common complications after angiographic procedures is contrast-induced nephropathy (CIN). As previously reported, the incidence of CIN is higher in patients undergoing cardiac procedures such as percutaneous coronary intervention (PCI) when compared to general population. Despite being the third etiology of hospital-acquired acute renal failure, permanent renal impairment is quite rare and the progression of CIN is often modest [1]. Ultimately, most CIN patients did not require permanent hemodialysis. However, CIN is yet found to be associated with longer length of hospitalization with substantial financial burden, increasing disability and in-hospital mortality $[2,3]$.

To date, the specific therapeutic intervention for post-procedural CIN is still inconclusive. Therefore, the mainstay of CIN management, other than supportive therapy, lies within preventive strategies and adequate risk-benefit assessment. Several risk scoring systems were developed to assess the risk of CIN occurrence in patients undergoing radiographic procedure requiring administration of iodinated contrast. One of the most cited scorings for the prediction of CIN in patients undergoing PCI is the Mehran risk score (MRS) $[4,5]$.

The score includes eight readily available clinical parameters which altogether could stratify patients into four risk 
groups (low, moderate, high and very high risk of CIN).

However, there were some limitations concerning the implication of MRS. Firstly, patients who were treated with PCI for acute myocardial infarction were excluded from score development dataset, which in turn limited generalizability to this specific subset of patients. Secondly, in prognostication of CIN, eight clinical variables were required. This was considered by many physicians as excessive and impractical for routine use.

This study intended to develop a new risk stratification score which is simpler and more practical than the MRS with comparable performance for the prediction of CIN after PCI in patients with acute ST-segment elevation myocardial infarction (STEMI).

\section{Materials and Methods}

\section{Study design and setting}

A prognostic prediction research and clinical score development and validation study were conducted based on a singlecenter, retrospective cohort at the Central Chest Institute of Thailand (CCIT), a tertiary care medical center specializing in cardiopulmonary diseases administered by the Department of Medical Service, the Ministry of Public Health, Thailand. This study was registered on Thai Clinical Trials Registry (TCTR20190705001).

\section{Selection of participants}

Acute STEMI patients aged more than 18 years old who were treated with primary PCI at the Cardiac Catheterization Laboratory of the institute were included from June 1, 2017 to June 30, 2018. End-stage renal disease patients with estimated glomerular filtration less than $15 \mathrm{~mL} / \mathrm{min} / 1.73 \mathrm{~m}^{2}$ or being treated with peritoneal or hemodialysis, patients who were exposed to contrast media agent 7 days prior to the index PCI procedure, and patients who did not survive primary PCI were excluded from the study. The remaining patients constituted the derivation cohort for risk scoring development.

\section{Definition of CIN}

CIN was defined as the deterioration of renal function following the administration of contrast agents and unlikely to be explained by other possible causes. Patients with an absolute increase in serum creatinine of greater than $0.5 \mathrm{mg} / \mathrm{dL}$ or more than $25 \%$ from baseline value within $48 \mathrm{~h}$ after primary percutaneous coronary procedure were considered as CIN cases [6].

\section{Data collection}

Clinical characteristics and potential predictors were extracted from routinely collected medical records including age, gender, body surface area (BSA), atherosclerotic-associated comorbidi- ties such as hypertension, diabetes mellitus and hypercholesterolemia, history of previous myocardial infarction, history of previous stroke of transient ischemic attack, smoking history, alcohol consumption history, and prior medication use. Initial vital signs and laboratory values prior to PCI were also retrieved to justify each patient for having specific conditions as followed: congestive heart failure (CHF) was defined as patients with frank pulmonary edema or symptoms and signs consistent with New York Heart Association classification of severity of heart failure class III and IV at presentation to the hospital. According to the World Health Organization's criteria, anemic patients were defined as male patients with baseline hematocrit value of less than $39 \%$ and less than $36 \%$ for female patients [7]. Chronic kidney disease was defined as patients with baseline serum creatinine of more than $1.5 \mathrm{mg} / \mathrm{dL}$ or estimated glomerular filtration rate (eGFR) of less than 60 $\mathrm{mL} / \mathrm{min} / 1.73 \mathrm{~m}^{2}$. eGFR was calculated based on the formula proposed by Chronic Kidney Disease Epidemiology Collaboration (CKD-EPI).

All patients were evaluated with initial electrocardiogram (ECG) to identify possible walls of infarction and portable echocardiography to assess initial cardiac ejection fraction (EF), and wall motion abnormalities prior to endovascular procedure. Peri-procedural findings recorded included angiographic findings, contrast type, contrast volume used, type of coronary intervention done, and insertion of intra-aortic balloon pump (IABP). Hypotension was defined as patients with systolic blood pressure less than $80 \mathrm{~mm} \mathrm{Hg}$ for at least $1 \mathrm{~h}$ and subsequently required inotropic agents or insertion of IABP during and within $24 \mathrm{~h}$ range after percutaneous endovascular procedures [5]. The MRS consisting of eight clinical parameters was calculated for each patient.

\section{Statistical analysis and sample size calculation}

Continuous variables were summarized as mean and standard deviation (SD) and as number of frequency and percentages for categorical data. Comparisons between categorical variables were performed with Chi-square tests or Fisher's exact probability tests as appropriate.

To date, there was no generally recommended approach for sample size calculation in clinical risk score development. Thus, all available data in our database were used for score derivation to maximize statistical power and extend generalizability. As this study was intended to simplify and reduce the number of predictors from previously reported scoring system, the final number of predictors should be limited to avoid violation of the commonly suggested rule-of-thumb of 10 endpoint events per candidate parameter.

\section{Model development}

Eight initial predictors of CIN were chosen based on previously reported scoring system by Mehran et al [4, 5], which included hypotension, use of IABP, CHF, age $>75$ years, anemia, diabetes mellitus, contrast media volume, and chronic kidney disease. This study also included five additional poten- 
tial predictors for model development, which were EF, walls of infarction from ECG, type of contrast media, angiographic findings, and type of PCI done for patients. All 13 predictors were classified into two subgroups: clinical characteristics and peri-procedural characteristics.

Exploratory analysis was done for all potential predictors using univariable logistic regression. Odds ratio (OR) with its $\mathrm{P}$ value and area under the receiver operating characteristic curve (AuROC) with its 95\% confidence interval (CI) was reported separately for each predictor variable. Multivariable logistic regression analysis was then performed to identify independent predictor of CIN. The removal of non-contributing predictors was based primarily on clinical relevancy and statistical significance. Predictor variables with OR closest to 1.00 and insignificant $\mathrm{P}$ value of $>0.1$ were sequentially eliminated from logistic regression model.

The reduced multivariable model was evaluated for its predictive performance in terms of discrimination and calibration. Measure of discrimination was reported as AuROC. Measure of calibration was reported as Hosmer-Lemeshow goodness of fit statistics.

\section{Score derivation and validation}

Each final predictor was assigned with specific score based on each item's logistic regression coefficient. The logistic coefficient of each predictor was divided by the lowest coefficient in the model and subsequently rounded up to the nearest nondecimal integer for forecasted applicability. The sum total score was then further categorized based on baseline CIN risk as being at lower/equivocal or higher risk to the total STEMI patients within the study. Positive predictive value (PPV) was calculated for each score category to indicate average risk of the patients. Measures of calibration and discrimination were also performed via regression of CIN on the score model. Calibration plot comparing score predicted risk versus observed risk was presented. Predictive performance was validated and compared between newly derived risk score and the MRS by non-parametric ROC regression with 1,000 replicates bootstrapped sampling. A P value of $<0.05$ was considered to indicate statistical significance.

\section{Statement of ethics}

All patient data were extracted from medical record retrospectively and were finally kept in research archives. The study protocol was approved by the Ethical Committee of Central Chest Institute, Department of Medical Services, Ministry of Public Health, Thailand.

\section{Results}

\section{Participants}

A total of 217 patients, 43 with CIN and 174 without CIN,

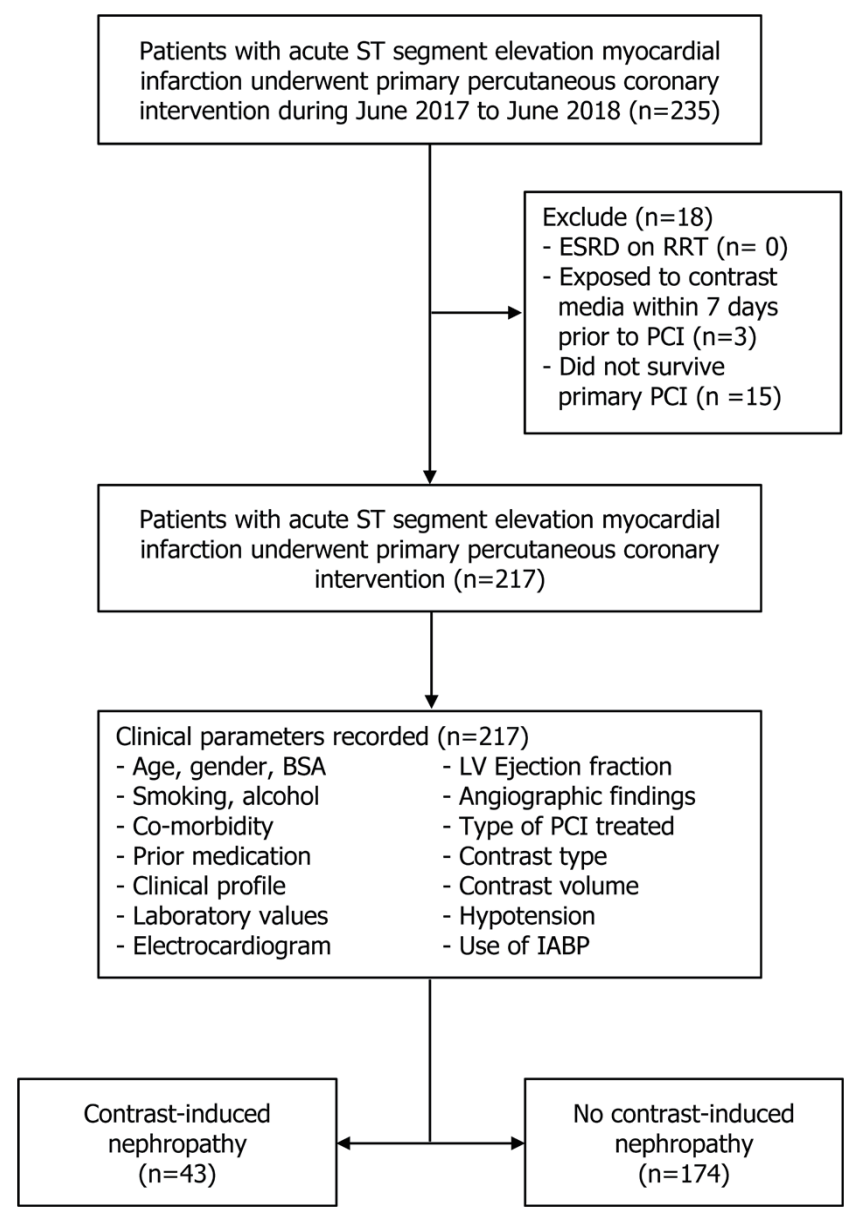

Figure 1. Flow of patients within the study.

were included for analysis (Fig. 1). The incidence of CIN in the study cohort was $19.8 \%$. Baseline clinical characteristics and peri-procedural findings were presented in Tables 1 and 2. Patients with CIN, when compared to those without CIN, were older $(66.4 \pm 12.3$ vs. $59.6 \pm 12.0$ years old, $\mathrm{P}=0.001)$, were anemic $(39.5 \%$ vs. $22.4 \%, \mathrm{P}=0.024)$, had lower systolic blood pressure $(103.3 \pm 30.9$ vs. $124.1 \pm 27.5 \mathrm{~mm} \mathrm{Hg}, \mathrm{P}$ $<0.001)$, had lower diastolic blood pressure $(60.7 \pm 19.1$ vs. $74.4 \pm 16.3 \mathrm{~mm} \mathrm{Hg}, \mathrm{P}<0.001)$, had higher heart rate $(90.7$ \pm 25.3 vs. $81.2 \pm 17.4 \mathrm{bpm}, \mathrm{P}=0.005)$, had higher incidence of acute heart failure at presentation $(51.2 \%$ vs. $10.3 \%, \mathrm{P}<$ $0.001)$, had poorer left ventricular EF of less than $40 \%(69.8 \%$ vs. $22.4 \%, \mathrm{P}<0.001)$, had lower eGFR $(68.3 \pm 26.7$ vs. $81.3 \pm$ $\left.25.5 \mathrm{~mL} / \mathrm{min} / 1.73 \mathrm{~m}^{2}, \mathrm{P}=0.004\right)$, had lower hematocrit level $(38.6 \pm 7.2 \%$ vs. $41.3 \pm 5.6 \%, \mathrm{P}=0.010)$, had higher serum cholesterol level (224.6 \pm 75.1 vs. $195.0 \pm 49.6 \mathrm{mg} / \mathrm{dL}, \mathrm{P}=0.004)$, had higher low-density lipoprotein (LDL) level (151.7 \pm 56.0 vs. $127.7 \pm 40.8 \mathrm{mg} / \mathrm{dL}, \mathrm{P}=0.002)$, had higher percentage of triple-vessel disease (62.8\% vs. $31.0 \%, \mathrm{P}=0.003)$, had higher incidence of peri-procedural hypotension $(55.8 \%$ vs. $12.6 \%, \mathrm{P}$ $<0.001)$, and had higher incidence of IABP insertion $(44.2 \%$ vs. $4.6 \%, \mathrm{P}<0.001)$. Clinical parameters with high predictive performance as measured by AuROC of more than 0.70 from univariable logistic regression were heart failure at presenta- 
Table 1. Clinical Characteristics

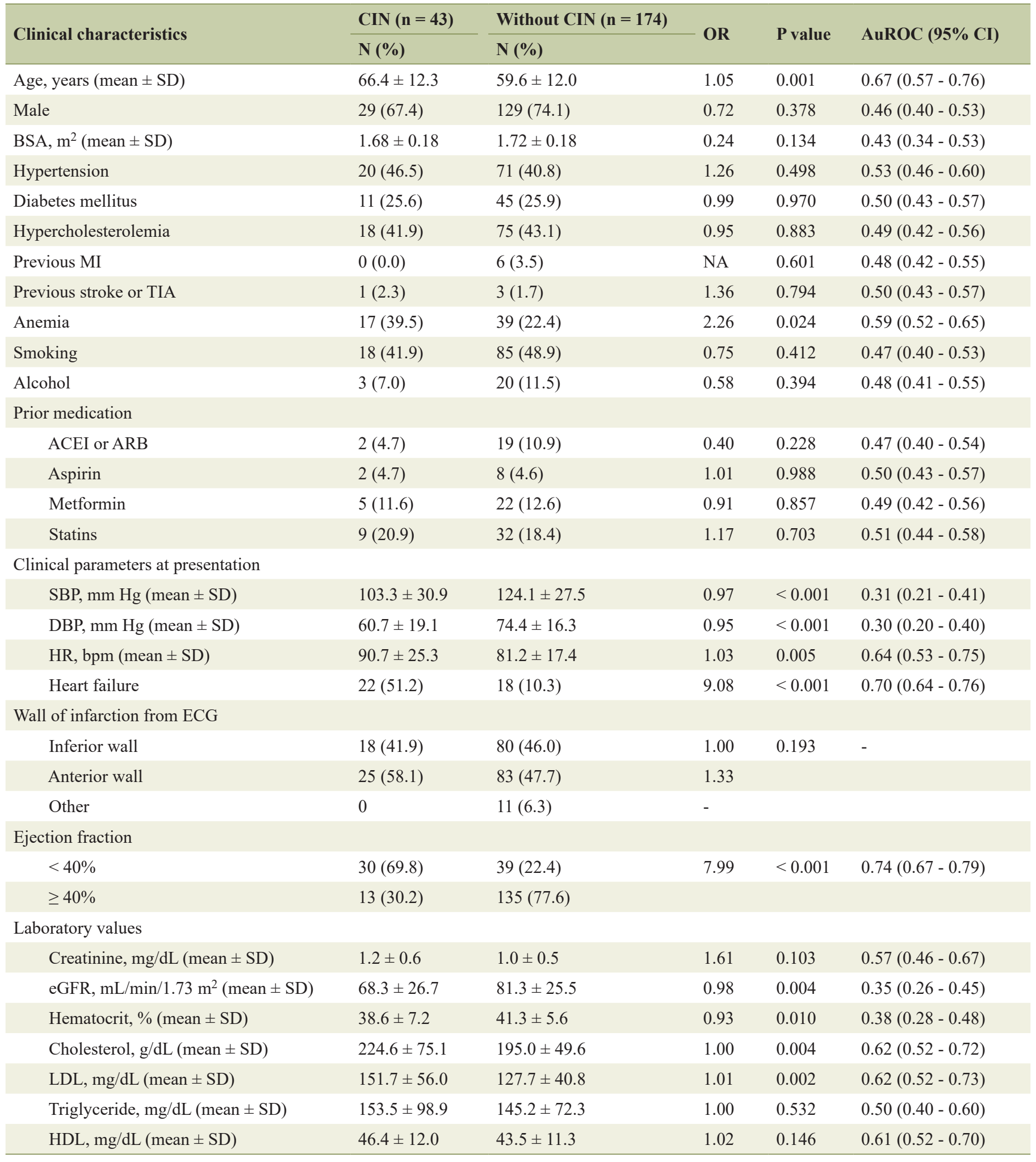

CIN: contrast-induced nephropathy; SD: standard deviation; OR: odds ratio; AuROC: area under the receiver operating characteristic curve; MI: myocardial infarction; TIA: transient ischemic attack; BSA: body surface area; ACEI: angiotensin-converting enzyme inhibitor; ARB: angiotensin receptor blocker; SBP: systolic blood pressure; DBP: diastolic blood pressure; HR: heart rate; ECG: electrocardiogram; eGFR: estimated glomerular filtration rate; LDL: low-density lipoprotein; HDL: high-density lipoprotein; NA: not applicable. 
Table 2. Operative Procedure and Findings

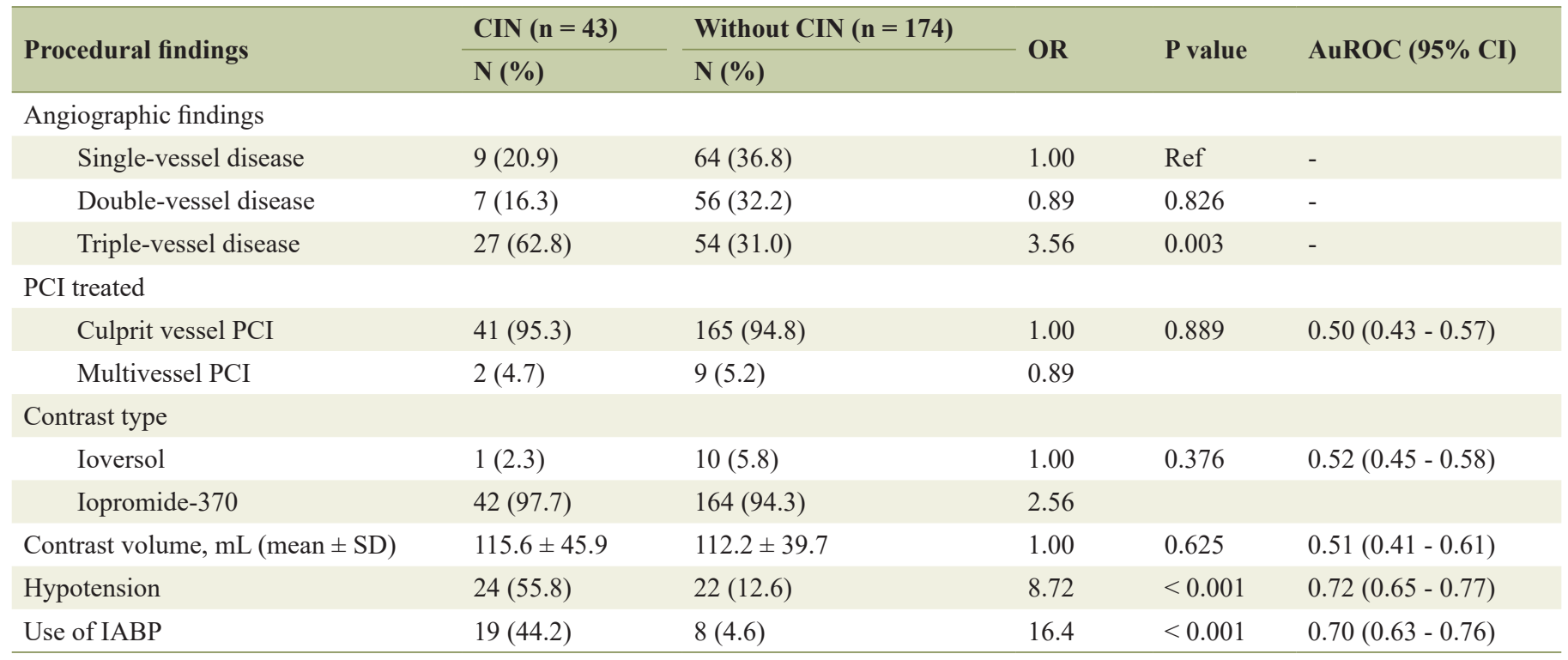

CIN: contrast-induced nephropathy; OR: odds ratio; AuROC: area under the receiver operating characteristic curve; CI: confidence interval; IABP: intra-aortic balloon pump; $\mathrm{PCl}$ : percutaneous coronary intervention; SD: standard deviation.

tion, EF less than $40 \%$, peri-procedural hypotension and use of IABP. In this study, none of the patients required acute hemodialysis after PCI.

\section{Model development and validation}

Thirteen potential clinical predictors were simultaneously explored under multivariable logistic regression (Table 3). Statistically significant predictors with $\mathrm{P}$ value of less than 0.100 were patients aged more than 75 years old, anterior wall infarction from ECG, EF less than 40\%, triple-vessel disease from angiographic findings, and use of IABP. After sequential elimination of non-contributive and non-significant predictors, three independent predictors (EF of less than $40 \%$, triplevessel disease as findings from angiogram, and use of IABP) were left in the final logistic model. The logit coefficient of each predictor was used as a weight for score transformation. A weighted score was assigned to each predictor as followed: 1 point for patients whose angiographic finding shows triplevessel disease, 2 points for patients whose initial left ventricular EF was less than $40 \%$, and 3 points for patients for whom

Table 3. Multivariable Logistic Regression Analysis

\begin{tabular}{llll}
\hline Variables & OR & 95\% CI & P value \\
\hline Age $>$ 75 years & 3.40 & $1.01-11.45$ & 0.048 \\
Diabetes mellitus & 0.85 & $0.32-2.28$ & 0.748 \\
Anemia & 1.30 & $0.46-3.73$ & 0.621 \\
Hypotension & 2.85 & $0.77-10.58$ & 0.117 \\
CHF & 1.04 & $0.28-3.94$ & 0.949 \\
Anterior wall infarction from ECG & 2.49 & $0.99-6.24$ & 0.052 \\
Ejection fraction $<40 \%$ & 4.87 & $1.92-12.35$ & 0.001 \\
eGFR $<60 \mathrm{~mL} /$ min/1.73 m ${ }^{2}$ & 0.54 & $0.18-1.60$ & 0.263 \\
Triple-vessel disease & 2.48 & $1.02-6.05$ & 0.046 \\
PCI treated & 0.19 & $0.02-2.08$ & 0.175 \\
Iopromide 370 (contrast media) & 8.82 & $0.62-124.96$ & 0.107 \\
Contrast media volume (every $100 \mathrm{~mL}$ increment) & 1.00 & $0.99-1.01$ & 0.940 \\
Use of IABP & 4.55 & $0.87-23.68$ & 0.072 \\
\hline
\end{tabular}

OR: odds ratio; $\mathrm{Cl}$ : confidence interval; ECG: electrocardiogram; IABP: intra-aortic balloon pump; CHF: congestive heart failure; eGFR: estimated glomerular filtration rate; TVD: triple-vessel disease; PCI: percutaneous coronary intervention. 
Table 4. Best Multivariable Clinical Predictors, OR, $95 \% \mathrm{Cl}$, Logistic Regression Beta Coefficient $(\beta)$ and Assigned Item Scores

\begin{tabular}{|c|c|c|c|c|c|}
\hline Predictors & OR & $95 \% \mathrm{CI}$ & P value & $\beta$ & Score \\
\hline Ejection fraction $<40 \%$ & 4.47 & $1.94-10.35$ & 0.001 & 1.50 & 2 \\
\hline Triple-vessel disease & 2.80 & $1.25-6.27$ & 0.012 & 1.03 & 1 \\
\hline Constant & 0.05 & $0.02-0.11$ & & -2.91 & \\
\hline
\end{tabular}

OR: odds ratio; $\mathrm{Cl}$ : confidence interval; IABP: intra-aortic balloon pump.

IABP was inserted.

The newly derived risk stratification score was named after the Central Chest Institute of Thailand or CCIT. The CCIT score ranged from a minimum of 0 point to a maximum of 6 points (Table 4). The score could predict the risk of CIN with good discriminative ability (AuROC: $0.83,95 \%$ CI: 0.76 - 0.90) which was higher when compared to that of the MRS (AuROC: 0.78, 95\% CI: 0.69 - 0.87) (Fig. 2). The difference of AuROC between the two scoring systems was borderline significant $(\mathrm{P}=0.082)$. Measures of calibration were visualized through calibration plot which showed that the score predicted risk of CIN and the observed risk of CIN in the derivation cohort concomitantly increased (Fig. 3). Hosmer-Lemeshow goodness of fit statistics also showed non-significant $\mathrm{P}$ of 0.883 . We performed internal validation of the score via nonparametric ROC with 1,000 bootstrap sampling, the results was acceptable predictive performance (AuROC: 0.77, 95\% CI: $0.68-0.85)$.

The CCIT score predicted range of CIN occurrence probability from $6.0 \%$ to $81.7 \%$. As the baseline risk in this cohort was $19.8 \%$, the score was categorized into low-risk and high-risk groups at the cut-off point of 2, for which the score-predicted risk equals to the baseline CIN risk. Patients with CCIT score lower or equal to 2 would be classified as low-risk patients (PPV: 9.9, 95\% CI: 5.4 - 14.4) while patients with higher CCIT score (3 or more) would be classified as high-risk patients (PPV: 56.5, 95\% CI: 42.4 - 70.8) (Table 5).

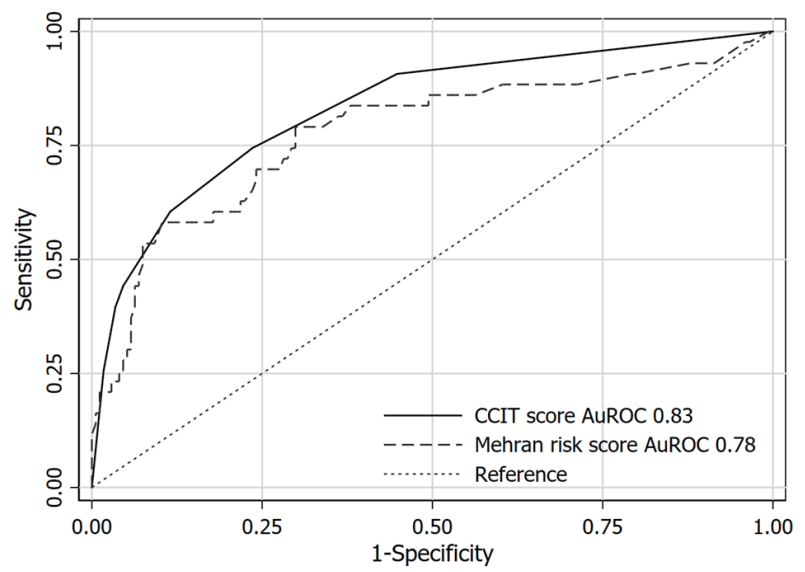

Figure 2. Comparison of area under receiver operating characteristic curve (AuROC) between new CCIT score and the Mehran risk score (MRS) in discriminating CIN cases. CCIT: Central Chest Institute of Thailand; CIN: contrast-induced nephropathy.

\section{Discussion}

According to the Thai Acute Coronary Syndrome Registry in $2007,40 \%$ of patients with coronary artery disease showed STsegment elevation in initial ECG. Acute STEMI is acute lifethreatening conditions which could finally lead to a high inhospital mortality rate of $17 \%[8,9]$. STEMI causes extensive damage to myocardium and acute reduction in EF which leads to hemodynamic instability and medullary ischemia. Thus, this group of patients is considered as higher risk of developing CIN $[10,11]$. In this current cohort study, the CIN incidence was $19.8 \%$ which was significantly higher than that in patients undergoing elective PCI at 1-3\% [12], but consistent to the number in patients requiring emergency PCI at 10-16\% [13, 14$]$.

The MRS consists of eight potential predictors which could accurately predict CIN occurrence in patients undergoing PCI, but the score was based on only non-emergency PCI patients. Acute myocardial infarction patients were excluded from the derivation cohort, limiting the generalizability for application to this specific group of high-risk patients. It is possible that a different set of prognostic factors is needed to prognosticate CIN occurrence within this domain of patients. In our study, three potential predictors of CIN in acute STEMI patients were identified, which were EF of less than $40 \%$, triple-vessel disease from angiogram and the use of IABP.

IABP insertion was the strongest predictor within CCIT score and the only predictor that was consistent with the MRS. IABP was performed after PCI mostly in patients who developed hypotension or cardiogenic shock in peri-proce-

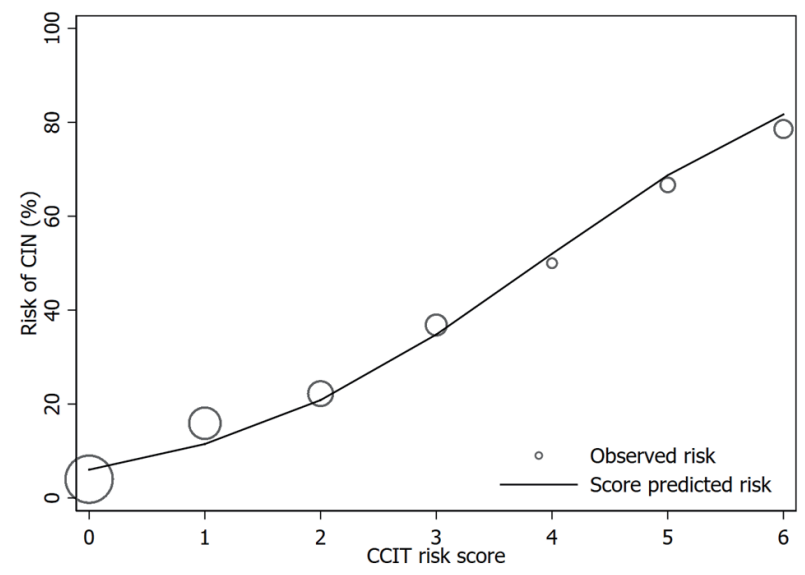

Figure 3. Calibration plot of score predicted risk vs. observed risk of $\mathrm{CIN}$ cases. CIN: contrast-induced nephropathy. 
Table 5. Distribution of Contrast-Induced Nephropathy Cases Across Two Different Categories of CCIT Score (Low Risk and High Risk for CIN)

\begin{tabular}{|c|c|c|c|c|c|c|c|c|}
\hline \multirow{2}{*}{ Risk categories } & \multirow{2}{*}{ Score } & \multicolumn{2}{|c|}{$\operatorname{CIN}(n=43)$} & \multicolumn{2}{|c|}{ Without CIN $(n=174)$} & \multirow{2}{*}{ PPV } & \multirow{2}{*}{$95 \%$ CI } & \multirow{2}{*}{ Pvalue } \\
\hline & & $\mathbf{N}(\%$ & & $\mathbf{N}(\%$ & & & & \\
\hline Low & $0-2$ & 17 & 39.5 & 154 & 88.5 & 9.9 & $5.4-14.4$ & $<0.001$ \\
\hline Mean $\pm \mathrm{SE}$ & & 3.3 & \pm 0.3 & 0.9 & \pm 0.1 & & & $<0.001$ \\
\hline
\end{tabular}

CCIT: Central Chest Institute of Thailand; CIN: contrast-induced nephropathy; PPV: positive predictive value; Cl: confidence interval; SE: standard error.

dural period. Thus, the use of IABP is a marker of significant hemodynamic disturbance or severe atherosclerosis without hypotension [5]. Patients with multivessel involvement and proximal left anterior descending (LAD) disease are at greater risk of developing CIN attributed to damaged myocardium and impaired left ventricular EF which leads to hemodynamic instability and impaired renal blood flow [15]. Impaired or reduced left ventricular EF had been identified as an important risk factor of CIN in previous studies $[16,17]$, and also found to be related to long-term mortality rate after cardiac catheterization [18].

The only one factor from the Mehran study remained in our model was the insertion of IABP, while the other two factors were newly identified in our study. Other Mehran's major predictors such as hypotension, $\mathrm{CHF}$, chronic kidney disease (defined by eGFR less than $<60 \mathrm{~mL} / \mathrm{min} / 1.73 \mathrm{~m}^{2}$ ) and age higher than 75 years were not present in our final model probably because of the following reasons: 1) Our score was derived in a much smaller cohort, with limited statistical power, it is possible that some independent predictors might not be included in our model; 2) The proportions of patients with CHF, chronic kidney disease and hypotension in this study were correlated to the proportion of patients who was inserted with IABP. With larger effect size from the use of IABP, the effect of the other two factors might be hindered; 3) The distribution of some predictors were unbalanced affecting the model's efficiency; 4) The percentage of triple-vessel disease or multivessel disease in our study was higher than in that in the Mehran study (37.3\% vs. 26.9\%) [5].

The MRS also found that contrast media volume was another independent predictor for CIN, while, in our study, no significant association was found. Our patient cohort used lesser contrast media volume compared to the Mehran study cohort $(112.9 \pm 40.9$ vs. $260.9 \pm 122.0 \mathrm{~mL})$ because our cardiac catheterization laboratory uses biplane angiography which helps us reduce the amount of contrast use. Based on 2018 European Society of Cardiology and the European Association for Cardiothoracic Surgery (ESC/EACTS) guidelines on myocardial revascularization, the risk of CIN significantly increases when the ratio of total contrast volume to GFR exceeds 3.7 [19]. In our study, only 12 patients were at increased risk of CIN from the use of higher volume of contrast alone.

The CCIT score was categorized into low and high risk at the cut-off points of 2 to be a clinical guidance on risk of CIN for clinicians. To date, the mainstay of CIN prevention still remains with adequate intravenous hydration. Other pre- ventive strategies did not have adequate scientific evidence to support their use in practice. In high-risk patients, the use of low-osmolar or iso-osmolar contrast media, minimization of the contrast volume during procedure, adequate pre and postprocedural hydration, and avoidance of nephrotoxic agents are recommended [5, 19-22].

This study carried some minor limitations. The derivation cohort included small number of patients and the data were retrospectively collected. The model was also based on only one center with high incidence of CIN. The score might not be suitable for use in other centers with lower incidence. Finally, the CCIT score should be externally validated in a larger, prospective study before being applied in real clinical practice.

In conclusion, this study proposed the new risk score based on three independent predictors. The CCIT score was proved to have good predictive value with fewer numbers of predictors, and could be practically applied for risk stratification of CIN in patients with acute STEMI who required emergent primary PCI.

\section{Acknowledgments}

The authors wish to acknowledge contribution of all the medical and nursing staff of the Cardiac Catheterization Laboratory, Cardiology Department at Central Chest Institute for their grateful collaborations.

\section{Financial Disclosure}

None to declare.

\section{Conflict of Interest}

The authors declared no potential conflict of interest with respect to the research, authorship, and/or publication of this article.

\section{Informed Consent}

All the data were retrospectively collected from routine cardiac catheterization laboratory database, thus informed consent 
was done as informed consent for cardiac catheterization procedure at the Central Chest Institute.

\section{Author Contributions}

Sukrisd Koowattanatianchai: principal project leader, conceived the study, initiated and participated in design and coordination, collected the data, analyzed the data, drafted the manuscript, read and approved the final manuscript. Thamarath Chantadansuwan: project advisor, participated in design, helped to draft the manuscript, read and approved the final manuscript. Akaphol Kaladee: participated in design, helped in data analysis and model development, helped to draft the manuscript, read and approved the final manuscript. Phichayut Phinyo: participated in design, helped in data analysis and model development, helped to draft the manuscript, read and approved the final manuscript. Jayanton Patumanond: participated in design, helped in data analysis and model development, helped to draft the manuscript, read and approved the final manuscript.

\section{References}

1. Nash K, Hafeez A, Hou S. Hospital-acquired renal insufficiency. Am J Kidney Dis. 2002;39(5):930-936.

2. Rihal CS, Textor SC, Grill DE, Berger PB, Ting HH, Best $\mathrm{PJ}$, Singh $\mathrm{M}$, et al. Incidence and prognostic importance of acute renal failure after percutaneous coronary intervention. Circulation. 2002;105(19):2259-2264.

3. Chen SL, Zhang J, Yei F, Zhu Z, Liu Z, Lin S, Chu J, et al. Clinical outcomes of contrast-induced nephropathy in patients undergoing percutaneous coronary intervention: a prospective, multicenter, randomized study to analyze the effect of hydration and acetylcysteine. Int J Cardiol. 2008;126(3):407-413.

4. Halkin A, Singh M, Nikolsky E, Grines CL, Tcheng JE, Garcia E, Cox DA, et al. Prediction of mortality after primary percutaneous coronary intervention for acute myocardial infarction: the CADILLAC risk score. J Am Coll Cardiol. 2005;45(9):1397-1405.

5. Mehran R, Aymong ED, Nikolsky E, Lasic Z, Iakovou I, Fahy M, Mintz GS, et al. A simple risk score for prediction of contrast-induced nephropathy after percutaneous coronary intervention: development and initial validation. J Am Coll Cardiol. 2004;44(7):1393-1399.

6. Klein LW, Sheldon MW, Brinker J, Mixon TA, Skelding $\mathrm{K}$, Strunk AO, Tommaso CL, et al. The use of radiographic contrast media during PCI: a focused review: a position statement of the Society of Cardiovascular Angiography and Interventions. Catheter Cardiovasc Interv. 2009;74(5):728-746.

7. Dallman PR, Yip R, Johnson C. Prevalence and causes of anemia in the United States, 1976 to 1980. Am J Clin Nutr. 1984;39(3):437-445.

8. Srimahachota S, Kanjanavanit R, Boonyaratavej S, Boonsom W, Veerakul G, Tresukosol D, for the TACSR Group. Demographic, management practices and in-hospital out- comes of Thai Acute Coronary Syndrome Registry (TACSR): the difference from the Western world. J Med Assoc Thai. 2007;90(Suppl 1):1-11.

9. Sanguanwong S, Srimahachota S, Tungsubutra W, Srichaiveth B, Kiatchoosakun S. Predictors of in-hospital mortality in Thai STEMI patients: results from TACSR. J Med Assoc Thai. 2007;90(Suppl 1):91-97.

10. McCullough PA, Wolyn R, Rocher LL, Levin RN, O'Neill WW. Acute renal failure after coronary intervention: incidence, risk factors, and relationship to mortality. Am J Med. 1997;103(5):368-375.

11. Marenzi G, Lauri G, Assanelli E, Campodonico J, De Metrio M, Marana I, Grazi M, et al. Contrast-induced nephropathy in patients undergoing primary angioplasty for acute myocardial infarction. J Am Coll Cardiol. 2004;44(9):1780-1785.

12. Mehran R, Nikolsky E. Contrast-induced nephropathy: definition, epidemiology, and patients at risk. Kidney Int Suppl. 2006;100:S11-15.

13. Narula A, Mehran R, Weisz G, Dangas GD, Yu J, Genereux P, Nikolsky E, et al. Contrast-induced acute kidney injury after primary percutaneous coronary intervention: results from the HORIZONS-AMI substudy. Eur Heart J. 2014;35(23):1533-1540.

14. Shacham Y, Leshem-Rubinow E, Gal-Oz A, Arbel Y, Keren G, Roth A, Steinvil A. Acute cardio-renal syndrome as a cause for renal deterioration among myocardial infarction patients treated with primary percutaneous intervention. Can J Cardiol. 2015;31(10):1240-1244.

15. Suresh H, Anup H. Study of risk factors for contrast-induced nephropathy in patients with coronary artery disease. J Evid Based Med Healthc. 2017;4(17):979-982.

16. Caruso M, Balasus F, Incalcaterra E, Ruggieri A, Evola $\mathrm{S}$, Fattouch K, Bracale UM, et al. Contrast-induced nephropathy after percutaneous coronary intervention in simple lesions: risk factors and incidence are affected by the definition utilized. Intern Med. 2011;50(9):983-989.

17. Chong E, Poh KK, Liang S, Tan HC. Risk factors and clinical outcomes for contrast-induced nephropathy after percutaneous coronary intervention in patients with normal serum creatinine. Ann Acad Med Singapore. 2010;39(5):374-380.

18. Wang K, Li HL, Bei WJ, Guo XS, Chen SQ, Islam SMS, Chen JY, et al. Association of left ventricular ejection fraction with contrast-induced nephropathy and mortality following coronary angiography or intervention in patients with heart failure. Ther Clin Risk Manag. 2017;13:887895.

19. Neumann FJ, Sousa-Uva M, Ahlsson A, Alfonso F, Banning AP, Benedetto U, Byrne RA, et al. 2018 ESC/ EACTS Guidelines on myocardial revascularization. Eur Heart J. 2019;40(2):87-165.

20. Kern MJ. SAH. SCAI Interventional Cardiology Review. 3rd ed. Lippincott Williams \& Wilkins; 2018.

21. Murphy SW, Barrett BJ, Parfrey PS. Contrast nephropathy. J Am Soc Nephrol. 2000;11(1):177-182.

22. Barrett BJ. Contrast nephrotoxicity. J Am Soc Nephrol. 1994;5(2):125-137. 